\title{
Uma experiência de orientação de pesquisas científicas nos anos finais do Ensino Fundamental do Colégio de Aplicação da UFRGS
}

\section{Una experiencia de orientación de investigaciones científicas en los últimos años de la primaria en el Colégio de Aplicação de UFRGS}

\author{
Victor Hugo Nedel Oliveira victor.juventudes@gmail.com \\ Universida de Federal do Rio Grande do Sul
}

\begin{abstract}
Resumo: A prática pedagógica da iniciação científica na escola básica vem ganhando relevância na contemporaneidade, na medida em que a construção do co nhecimento perpassa os processos de buscas por respostas de questionamentos feitos pelos estudantes a partir de suas experiências. O principal objetivo do presente texto é relatar o desenvolvimento de uma experiência de orientação de pesquisa científica no âmbito da escola, a partir da descrição das etapas envolvidas das investigações de estudantes pesquisadores do ensino fundamental do Colégio de Aplicação da UFRGS. Para tanto, verificou-se o papel do docente orientador e de seis estudantes na disciplina de Iniciação Científica, formando o corpus da investigação. Considera-se, portanto, que a ação discutida possibilita entender os estudantes como protagonistas de suas aprendizagens, a partir do emprego da Iniciação Científica na escola básica. Ao mesmo tempo, o processo do exercício reflexivo entre as atividades dos estudantes e do orientador coloca em ação as perspectivas de uma práxis docente conectada com as exigências contemporâneas.
\end{abstract}

Palavras-chave: iniciação científica; palavra escola básica; orientação de pesquisas.

Resumen: La práctica pedagógica de la iniciación científica en la escuela básica ha ido ganando relevancia en la época contemporánea, a medida que la construcción del conocimiento pasa por los procesos de búsqueda de respuestas a las preguntas que hacen los estudiantes a partir de sus experiencias. El objetivo principal de este tex to es reportar el desarrollo de una experiencia de orientación a la investigación científica dentro de la escuela, a partir de la descripción de las etapas involucradas en las investigaciones de los estudiantes-investigadores de la escuela primaria del Colégio de Aplicação de UFRGS. Para ello, se verificó el rol del docente asesor y seis estu diantes en la asignatura de Iniciación Científica, conformando el corpus de la investigación. Por tanto, se considera que la acción discutida permite entender a los estudiantes como protagonistas de su aprendizaje, a partir del uso de la Iniciación Científica en la escuela básica. Al mismo tiempo, el proceso de ejercicio reflexivo entre las actividades de los estudiantes y el asesor pone en práctica las perspectivas de una praxis docente conectada con las demandas contemporáneas.

Palabras clave: iniciación científica; escuela básica; orientación de investigaciones.

Recebido em: 30/04/ 2020

Aceito em: 19/11/2020 


\section{PARA INÍCIO DE CONVERSA}

A pesquisa escolar vem se constituindo como importante estratégia de en sinoaprendizagem no contemporâneo. Pesquisadores como Bernardes e Fernandes (2002), Fialho (2013), Vasques e Oliveira (2020a; 2020b) já destacaram em suas produçõ es os benefícios da utilização das práticas de pesquisa escolar no âmbito da escola básica.

Este é um relato que busca apresentar uma experiência de orientação de pesquisa científica escolar, desenvolvida durante o segundo semestre letivo do ano de 2018, com alunos dos $6^{\circ}$ e do $7^{\circ}$ anos do Ensino Fundamental, matriculados no Colégio de Aplicação da Universidade Federal do Rio Grande do Sul. Pensar a constituição de ciência para esses estudantes (OLIVEIRA; VASQUES, 2020), portanto também é entender como tais sujeitos percebem os processos científicos em seus cotidianos.

Para que se aclare, a constituição metodológica de orientação de pesquisa científica escolar foi organizada a partir da bricolagem de algumas estratégias que já vinham sido utilizadas no Projeto relativo às séries em curso e outras estratégias que foram adotadas pelo orientador em específico.

Adotou-se um conjunto de estratégias, inicialmente divididas em quatro fases (figura 1), que compõem um circuito integrado de atividades, de maneira que quando da finalização de um momento, possa dar início a outros momentos, haja vista que a pesquisa científica possibilita a abertura de janelas a novas possibilidades e novas dúvidas, perguntas e questionamentos.

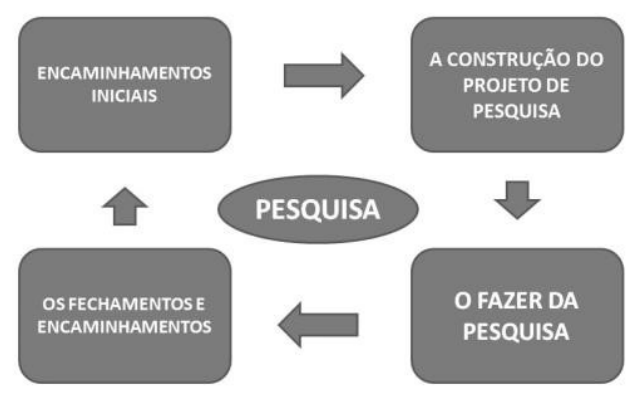

Figura 01: Esquema das fases da pesquisa. Orga nização: o a utor (2020)

Na sequência do texto serão apresentadas as quatro fases da pesquisa, a saber: encaminhamentos iniciais, a construção do projeto de pesquisa, o fazer da pesquisa, os fechamentos e encaminhamentos.

Recebido em: 30/04/2020

Aceito em: 19/11/2020 


\section{ANDAMENTOS METODOLÓGICOS DAS INVESTIGAÇÕES ORIENTADAS}

\subsection{OS ENCAMINHAMENTOS INICIAIS}

a) Atividades disparadoras para escolha e delimitação do tema de pesquisa

Inicialmente, algumas atividades disparadoras possibilitaram a escolha e a delimitação do tema de pesquisa. Em um primeiro momento, os alunos escreveram em uma folha seus interesses de pesquisa, anotando o tema de pesquisa, uma pergunta principal, três palavras-chave (iniciais). O grupo de professores envolvidos no proces so realizou intervenções nos escritos dos alunos com os mesmos, de maneira a realizar uma primeira refinada na escolha do tema de pesquisa. A delimitação final foi organizada já com a divisão por grupos de pesquisa (vide próximo item) e com o auxílio do orientador designado para o grupo de pesquisa.

b) A constituição do grupo de pesquisa

O grupo de pesquisa se constituiu a partir da afinidade de temas optados e indicados pelos alunos. A escolha do orientador para o grupo de pesquisa foi organizada a partir da afinidade de formação do professor com o tema geral do grupo de pe squisa. Apresentam-se os sujeitos da pesquisa não como os pesquisados, mas na qualidade de pesquisadores, visto que sua atuação enquanto estudantes-pesquisadores os colocaram na condição de investigados deste artigo. O quadro 1 apresenta a sigla atribuíd a a cada sujeito, de maneira a preservar a identidade de cada um; o sexo; a idade e o ano escolar dos sujeitos.

Quadro 1 - Apresentação dos sujeitos pesquisadores. Orga nização: o a utor (2020)

\begin{tabular}{|c|c|c|c|}
\hline Sigla & Sexo & Idade & Ano Escolar \\
\hline S1 & M & 13 & $7^{\mathbf{0}}$ ANO \\
\hline S2 & F & 12 & $6^{\mathbf{0}}$ ANO \\
\hline S3 & M & 12 & $7^{\mathbf{0}}$ ANO \\
\hline S4 & M & 12 & $7^{\mathbf{0}}$ ANO \\
\hline S5 & M & 14 & $7^{\mathbf{0}}$ ANO \\
\hline S6 & F & 12 & $7^{\mathbf{0}}$ ANO \\
\hline S7 & F & 13 & $7^{\mathbf{0}}$ ANO \\
\hline
\end{tabular}


Edição Especial: XVI Encontro sobre Investigação na Escola - EIE

ISSN: 2595-4520 Vol. 4, n. 2. 2021

O grupo de pesquisa, então constituído, foi denominado "Fenômenos Naturais", de maneira que melhor pudesse abranger os temas individuais dos alunos-sujeitospesquisadores e, à sua maneira, criar identidade do grupo.

\subsection{A CONSTRUÇÃO DO PROJETO E DA PESQUISA}

a) Construção das perguntas de pesquisa (pergunta principal e perguntas secundárias)

As perguntas de pesquisa (principal e secundárias) foram construídas a partir das discussões anteriores estabelecidas e da orientação coletiva e individual proporcionada pelo professor orientador. O quadro 2 apresenta a pergunta principal de pesquisa de cada sujeito pesquisador. Além destas perguntas, cada aluno teve a tarefa de organizar ao menos três perguntas secundárias de pesquisa, que complementem as dúvidas estabelecidas inicialmente na pergunta principal.

Quadro 2 - Pergunta principal de ca da pesquisa. Orga nização: o a utor(2020)

\begin{tabular}{|l|l|l|}
\hline Sujeito & \multicolumn{1}{|c|}{ Tema de Pesquisa } & \multicolumn{1}{c|}{ Pergunta Principal } \\
\hline S1 & Arroz & Como é feita a plantação do arroz? \\
\hline S2 & Buraco Negro & O que é o Buraco Negro? \\
\hline S3 & Buraco Negro & $\begin{array}{l}\text { O que aconteceria com o planeta Terra se } \\
\text { fosse engolido por um Buraco Negro? }\end{array}$ \\
\hline S4 & Extinção & O que pode nos levar a próxima extinção? \\
\hline
\end{tabular}

Nota-se, por exemplo, a distinção na formulação da pergunta de pesquis a en tre os sujeitos S2 e S3, que apresentaram a mesma temática para suas investigações. A complexidade na elaboração da pergunta do sujeito S3 pode ser analis ada a partir das experiências anteriores realizadas, na medida em que esta é a quarta experiência deste sujeito, em contrapartida de ser a primeira experiência do sujeito S2 (vide quadro 1). Entende-se que, cada um em seu tempo (BORGES; LIMA, 2020), desenvolve as estratégias de avanço em suas práticas de investigação e que a maturidade a partir da própria idade colabora neste processo de aperfeiçoamento ao pensar sua pesquisa científica escolar.

b) As palavras-chave e os conceitos de pesquisa 
Edição Especial: XVI Encontro sobre Investigação na Escola - EIE

ISSN: $2595-4520$

$$
\text { Vol. 4, n. 2. } 2021
$$

Após a construção das perguntas de pesquisa, retomaram-se as palavras-chave inicialmente organizadas pelos sujeitos pesquisadores, de maneira a organizar alguns conceitos centrais de cada pesquisa e, ainda, possibilitar a construção de uma visão ampla do processo que seria desenvolvido.

Quadro 3 - Pa la vras-chave de cada pesquisa. Orga nização: o a utor (2020)

\begin{tabular}{|l|l|l|}
\hline Sujeito & \multicolumn{1}{|c|}{ Tema } & \multicolumn{1}{c|}{ Palavras-chave } \\
\hline S1 & Arroz & Arroz; Agrotóxico; Plantado; Plantação; Banhado; Colheita. \\
\hline S4 & Extinção & $\begin{array}{l}\text { Extinção; Fim do mundo; Armas biológicas; Desastres naturais; } \\
\text { Mitos. }\end{array}$ \\
\hline S6 & Vulcões & Vulcão; Erupção; História; Brasil; Lava. \\
\hline S7 & Tornado & Tornado; Lugares; Maior; Pessoas; Clima. \\
\hline
\end{tabular}

As palavras-chave das pesquisas deram origem aos conceitos básicos de cada investigação e, ao mesmo tempo, serviram como ponto de partida para a confecção do primeiro mapa conceitual, tanto como palavra central quanto como palavras ramificadas.

c) O primeiro mapa conceitual

Para que se pudessem organizar as ideias prévias dos sujeitos-pesquisadores, confeccionou-se o primeiro mapa conceitual, no qual os alunos deveriam de colocar tudo o que conheciam em relação ao tema de pesquisa escolhido.

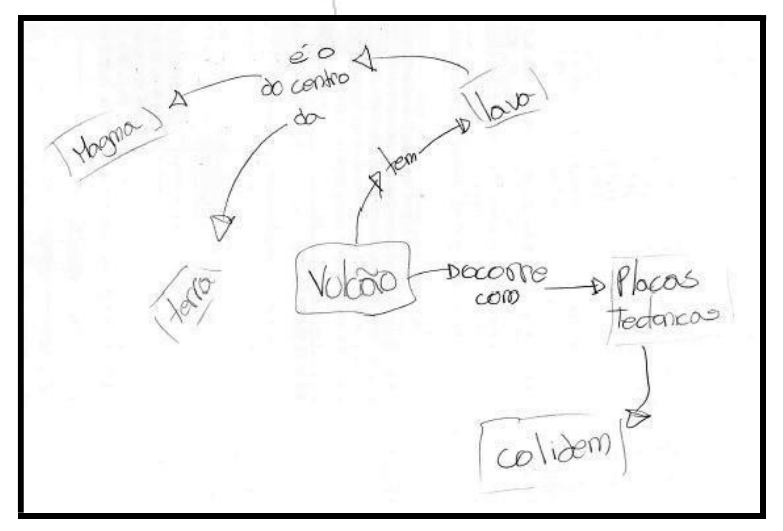

Figura 02: Primeiro mapa conceitual. Fonte: S6 (2018)

Fica claro que o primeiro mapa conceitual tendeu a ter poucas informações, visto que se tratou de um assunto ainda em fase inicial de exploração pelos pes quisadores. 
Edição Especial: XVI Encontro sobre Investigação na Escola - EIE

Encaminhando para as conclusões do tex to, será apresentado um comparativo evolutivo do primeiro para o segundo mapa conceitual.

d) Objetivos e relação com as perguntas de pesquisa

Para que se pudesse direcionar a pesquisa dos alunos de maneira exequível e que, ao mesmo tempo, tivessem objetivos que guiassem a pesquisa. Para tanto, a partir da pergunta principal e das perguntas secundárias, foram criados o objetivo principal e os objetivos secundários, respectivamente, como pode ser observado no quadro 4.

Quadro 4 - Quadro de rela ção entre os objetivos e as perguntas depesquisa. Fonte: S5 (2018)

\begin{tabular}{|l|l|}
\hline $\begin{array}{l}\text { OBJETIVO GERAL: Descobrir o } \\
\text { funcionamento da teoria das cordas. }\end{array}$ & $\begin{array}{l}\text { PERGUNTA PRINCIPAL: Como } \\
\text { funciona a teoria das cordas? }\end{array}$ \\
\hline $\begin{array}{l}\text { OBJETIVO ESPECÍFICO 1: Comparar o } \\
\text { antes e o depois da teoria das cordas. }\end{array}$ & $\begin{array}{l}\text { PERGUNTA SECUNDÁRIA 1: A } \\
\text { teoria das cordas mudou o mundo? }\end{array}$ \\
\hline $\begin{array}{l}\text { OBJETIVO ESPECÍFICO 2: Investigar quem } \\
\text { e quando a teoria das cordas foi criada. }\end{array}$ & $\begin{array}{l}\text { PERGUNTA SECUNDÁRIA 2: Quem } \\
\text { criou a teoria das cordas? }\end{array}$ \\
\hline
\end{tabular}

e) A justificativa

Várias foram as motivações apresentadas pelos jovens-pesquisadores para a escolha do tema de pesquisa de cada um. Apresentam-se as justificativas de cada sujeito:

Os meus tios são agricultores e então eu achei bacana pesquisar uma coisa sobre a agricultura e talvez seguir a tradição (S1).

$\mathrm{Eu}$ gostaria de estudar este tema porque caso a Terra fosse engolida por um Buraco negro adoraria saber o que aconteceria com o planeta (S3).

f) Procedimentos metodológicos

Os procedimentos metodológicos utilizados por todos os membros do grupo de pesquisa formado foram os mesmos, para que todos pudessem acompanhar as etapas metodológicas e seguir sob a orientação do professor nas etapas adequadas para as pesquisas. Inicialmente, foram realizadas pesquisas de levantamento bibliográfico na biblioteca do Colégio de Aplicação. Em dois momentos, foram realizadas pesquis as no laboratório de informática da escola, possibilitando que os sujeitos-pesquisadores 
Edição Especial: XVI Encontro sobre Investigação na Escola - EIE

ISSN: 2595-4520 Vol. 4, n. 2. 2021

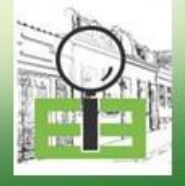

levantassem material de leitura sobre seus temas de pesquisa, mas que também buscassem imagens, assistissem vídeos e transpusessem as informações de suas investigações para a construção de um artigo científico. Dois mapas conceituais foram confeccionados, para que se avaliasse a evolução dos conhecimentos construídos a partir da construção da pesquisa científica em si. Ainda, foram realizados questionários com a comunidade escolar, a partir de levantamento no modelo da escala Likert (1932). Por fim, foram realizadas entrevistas com especialistas, que dessem conta de esclarec er tópicos que ainda não tinham sido trabalhados com os sujeitos pesquisadores.

g) O segundo mapa conceitual

Ao final das etapas de coleta e análise dos dados da pesquisa, os alunos construíram um segundo mapa conceitual. Todos avançaram em suas produções, c o mo pode ser observado nos dois mapas conceituais de S4, lado a lado, para que se possa ter noção do tamanho avanço conquistado pelo sujeito, a partir do processo da pesquisa científica escolar.

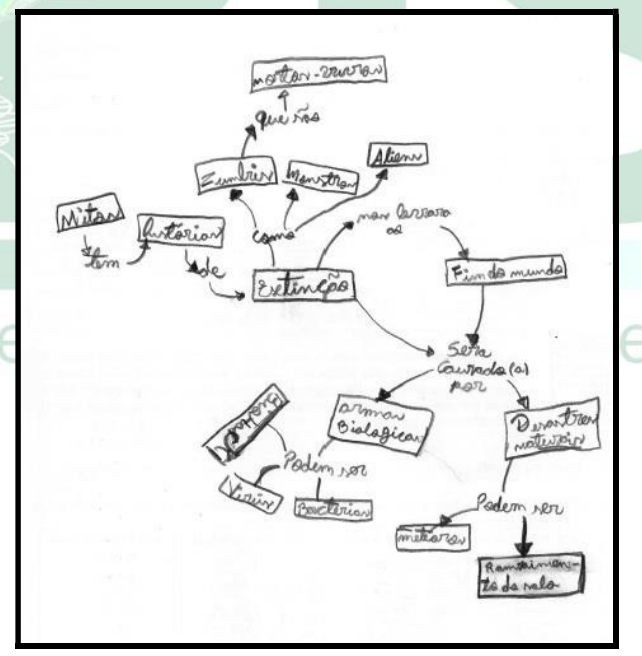

Figura 04: Exemplo do primeiro mapa conceitual. Fonte: S4 (2018)

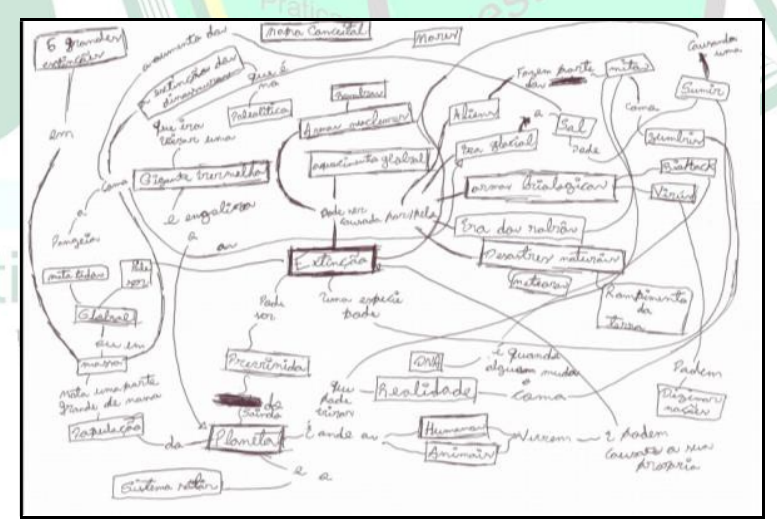

Figura 05: Exemplo do segundo mapa conceitual. Fonte: S4 (2018)

Nota-se o avanço no sentido de que S4 apresentou maior quantidade de palavraschave do primeiro mapa conceitual para o segundo mapa conceitual. O maior número de palavras-chave possibilitou que houvesse, também, um maior número de conexões entre os conceitos apresentados.

\subsection{OS FECHAMENTOS E ENCAMINHAMENTOS}

Recebido em: 30/04/2020

Aceito em: 19/11/2020 
Edição Especial: XVI Encontro sobre Investigação na Escola - EIE

ISSN: 2595- $4520 \quad$ Vol. 4, n. 2. 2021

a) Os aprendizados com a pesquisa

Ao perguntar aos jovens-sujeitos-pesquisadores sobre os aprendizados com a pesquisa, as respostas que vieram dizem respeito ao conteúdo, ou seja, as conclusões apresentadas com o desenvolvimento da pesquisa, através da coleta, análise e apresentação dos resultados.

b) Os principais aprendizados para além dos resultados

Perguntaram-se quais aprendizados o processo científico gerou nos alunos. Foram apresentadas respostas significativas, no sentido de que foi possível verificar que o movimento de pesquisa científica, com conteúdos delimitados, mobilizou conhecimentos e gerou aprendizagens significativas na vida dos sujeitos-pesquisadores.

Quadro 5 - Principa is a prendizados para a lém dos resultados. Organização: o a utor (2020)

\begin{tabular}{|l|l|}
\hline Sujeito & \multicolumn{1}{c|}{ Resposta } \\
\hline S5 & $\begin{array}{l}\text { É importante ter muita responsabilidade e muita dedicação pra pesquisar } \\
\text { sobre um assunto complexo como este, eu aprendi que eu sei me concentrar } \\
\text { muito só precisava ter conhecimento da minha dedicação e saber meu } \\
\text { conhecimento sobre as coisas. }\end{array}$ \\
\hline S6 & $\begin{array}{l}\text { Eu aprendi que sempre que tiver um Vulcão por muito tempo adormecido, } \\
\text { pode criar uma camada de rocha sobre a cratera, e no caso se um dia eu for } \\
\text { fazer uma escalada posso subir e na montanha e olhar a vista lá de cima. }\end{array}$ \\
\hline
\end{tabular}

c) A pesquisa científica no ensino fundamental: por que é importante?

Por fim, solicitou-se que os alunos respondessem a seguinte pergunta: "Por que é importante realizar a pesquisa científica na escola?". As respostas que vieram foram de extrema importância para avaliar tanto o processo desenvolvido neste relato, quanto reforçar o valor e necessidade do desenvolvimento de estratégias de pesquisa científica no ensino fundamental.

Quadro 6 - Por que é importante realizar a pesquisa científica na escola? Orga nização: o a utor (2020)

\begin{tabular}{|l|l|}
\hline Sujeito & Por que é importante realizar pesquisa científica na escola? \\
\hline S1 & $\begin{array}{l}\text { Nós aprendemos muitas coisas novas que não sabíamos e que estamos } \\
\text { tendo essa oportunidade de aprender. }\end{array}$ \\
\hline S3 & $\begin{array}{l}\text { Aprendemos coisas que não aprenderíamos com professores, pois eles } \\
\text { escolhem o assunto em projetos nós podemos escolher o que queremos } \\
\text { fazer. }\end{array}$ \\
\hline
\end{tabular}

\section{CONSIDERAÇÕES FINAIS}

Recebido em: 30/04/2020

Aceito em: 19/11/2020 
Edição Especial: XVI Encontro sobre Investigação na Escola - EIE

ISSN: 2595-4520 Vol. 4, n. 2. 2021

Seguramente, o processo de pesquisa científica na escola contribui significativamente para o crescimento dos conhecimentos produzidos pelos estudantes e pelos professores. A escola é, também, espaço de produção de conhecimentos e não apenas de reprodução dos mesmos, como fora sustentado por vários anos.

Práticas de orientação de pesquisa científica escolar corroboram, também, na construção de aprendizados significativos, visto que o que foi descrito pelos sujeitos pesquisadores enquanto da importância do fazer científico em sala de aula, reforça a necessidade da permanência deste recurso: os alunos escolhem seus temas de pesquisa; descobrem, por seus méritos, novos assuntos; conhecem habilidades importantes de trabalho e as sistematizam e reconhecem os esforços e aprendizagens criados e produzidos.

\section{REFERÊNCIAS}

BERNARDES, Alessandra Sexto; FERNANDES, Olívia Paiva. A pesquisa escolar em tempos de internet. Revista TEIAS: Rio de Janeiro, ano 3, nº 5, jan/jun 2002.

BORGES, Telma; LIMA, Valderez. A Educação pela Pesquisa como abordagem facilitadora da argumentação dialógica. Revista Insignare Scientia - RIS, v. 3, n. 3, 2020. Disponível em: https://periodicos.uffs.edu.br/index.php/RIS/article/view/11775 Acesso em: 20 nov. 2020.

FIALHO, Janaína. Experiência com estudantes de ensino médio através da pesquisa escolar orientada. Revista Perspectivas em Ciências da Informação, v. 18, n. 1, p. 15 25, jan./abr., 2013.

LIKERT, Rensis. A Technique for the Measurement of Attitudes. Archives of Psychology, 140: pp. 1-55, 1932.

OLIVEIRA, Victor Hugo Nedel; VASQUES, Daniel Giordani Percepção e representações Ciência de estudantes bolsistas de iniciação científica júnior. Revista Recebido em: 30/04/ 2020

Aceito em: $19 / 11 / 2020$ 
Edição Especial: XVI Encontro sobre Investigação na Escola - EIE

ISSN: 2595- $4520 \quad$ Vol. 4, n. 2. 2021

Ista Insignare Scientia

Mais,

V. $\quad 4$,

2020.

Disponível

em:

http://periodicos.ifsul.edu.br/index.php/educarmais/article/view/1991 Acesso em: 20 nov. 2020.

VASQUES, Daniel Giordani; OLIVEIRA, Victor Hugo Nedel. Iniciação científica na pandemia: uma análise dos estudos remotos ao ensino fundamental. Interfaces Científicas - Educação, v. 10, n. 1, 2020a. Disponível em: https://periodicos.set.edu.br/educacao/article/view/9084 Acesso em: 20 nov. 2020.

VASQUES, Daniel Giordani; OLIVEIRA, Victor Hugo Nedel. Iniciação científica na educação básica: estado do conhecimento a partir de artigos científicos de 2010 - 2020. Camine: Caminhos da Educação, v. 12, 2020b. Disponível em: https://ojs.franca.unesp.br/index.php/caminhos/article/viewFile/3247/2794 Ace ss o em: 20 nov. 2020. 